\title{
A novel universal algorithm for filament network tracing and cytoskeleton analysis
}

\author{
Daniel A. D. Flormann ${ }^{1,2} \quad$ Moritz Schu ${ }^{1} \quad$ Emmanuel Terriac ${ }^{2} \quad$ Divyendu Thalla $^{1,2}$ | \\ Lucina Kainka $^{1,2}$ | Marcus Koch ${ }^{2}$ | Annica K. B. Gad ${ }^{3,4}$ | Franziska Lautenschläger,
}

${ }^{1}$ Department of Physics, Saarland

University, Saarbruecken, Germany

${ }^{2}$ INM - Leibniz Institute for New Materials, Saarbruecken, Germany

${ }^{3}$ Department of Oncology and Metabolism, The Medical School, Weston Park Cancer Centre, Sheffield, UK

${ }^{4}$ Centro de Química da Madeira,

Universidade da Madeira, Funchal, Portugal

\section{Correspondence}

Daniel A. D. Flormann, Department of

Physics, Saarland University, Campus D2.2, 66123 Saarbruecken, Germany.

Email: Daniel.Flormann@web.de

\section{Funding information}

Leibniz Institute for New Materials (INM); Saarland University; DFG, Grant/Award Number: CRC 1027; Fundação para a Ciência e a Tecnologia (FCT); Portuguese Government, Grant/Award Number: PEstOE/QUI/UI0674/2013; Agência Regional para o Desenvolvimento da Investigaçaõ Tecnologia e Inovação (ARDITI); Centro de Química da Madeira, Grant/Award Number: M1420-01-0145-FEDER-000005; Universität des Saarlandes (US)

\begin{abstract}
The rapid development of advanced microscopy techniques over recent decades has significantly increased the quality of imaging and our understanding of subcellular structures, such as the organization of the filaments of the cytoskeleton using fluorescence and electron microscopy. However, these recent improvements in imaging techniques have not been matched by similar development of techniques for computational analysis of the images of filament networks that can now be obtained. Hence, for a wide range of applications, reliable computational analysis of such twodimensional methods remains challenging. Here, we present a new algorithm for tracing of filament networks. This software can extract many important parameters from grayscale images of filament networks, including the mesh hole size, and filament length and connectivity (also known as Coordination Number). In addition, the method allows sub-networks to be distinguished in two-dimensional images using intensity thresholding. We show that the algorithm can be used to analyze images of cytoskeleton networks obtained using different advanced microscopy methods. We have thus developed a new improved method for computational analysis of twodimensional images of filamentous networks that has wide applications for existing imaging techniques. The algorithm is available as open-source software.
\end{abstract}

\section{K E Y W O R D S}

actin, cytoskeleton, image analysis, intermediate filaments, microtubules

\section{1 | INTRODUCTION}

In recent decades, investigation of the filaments of the cytoskeleton, such as actin, vimentin, and microtubules, has become increasingly important for our understanding of cellular functions. ${ }^{1-4}$ For example, the spatial organization of the cytoskeletal network has an important role in cell migration, ${ }^{5,6}$ cancer metastasis, ${ }^{7,8}$ and cellular mechanics. ${ }^{1,9-12}$ The methods used to detect and image these structures vary from low-resolution fluorescence imaging, through high-resolution fluorescence imaging, to electron microscopy. ${ }^{3,6,13-16}$ The resolution of conventional light microscopy allows imaging

Abbreviations: FiNTA, filament network tracing algorithm; FM, fluorescence microscopy; SEM, scanning electron microscopy; STED, stimulated emission depletion; STORM, stochastic optical reconstruction microscopy.

Daniel A. D. Flormann and Moritz Schu contributed equally to this study.

[Correction added on June 11, 2022, after first online publication: Projekt DEAL funding statement has been added.]

This is an open access article under the terms of the Creative Commons Attribution-NonCommercial-NoDerivs License, which permits use and distribution in any medium, provided the original work is properly cited, the use is non-commercial and no modifications or adaptations are made.

() 2021 The Authors. The FASEB Journal published by Wiley Periodicals LLC on behalf of Federation of American Societies for Experimental Biology. 
down to $200 \mathrm{~nm}$, and super-resolution microscopy can now detect features with a resolution down to around $20 \mathrm{~nm}$. However, for the full structural networks, the resolution down to the atomic scale of electron microscopy is required.

The structures in these images can then be analyzed computationally, and the most commonly used method to investigate networks of grayscale imaging techniques are based on segmentation or pixel-based center lines, such as with ImageJ plugins; for example, DiameterJ and NeuronJ. ${ }^{17,18}$ These methods are user friendly and have well-defined and convenient output parameters. However, as the user has to define either the 'best segmented image' prior to the analysis or draw the starting and end points of each filament by hand, these methods rely upon the subjective analysis of the user. This thus reduces the reliability and reproducibility of these methods. More reliable analysis can be provided by vectorialbased algorithms that allow batch processing of grayscale network images. ${ }^{2,19}$ However, a limitation of this method is that the output parameters are commonly dedicated to a specific interest, and can therefore be limited.

To facilitate our ongoing analyses of scanning electron microscopy images of actin microfilament networks in cells, we have developed an algorithm that can analyze a wide range of filamentous networks that are imaged using different techniques, such as fluorescence microscopy, electron microscopy, and commercial photography techniques. We have named this algorithm the filament network-tracing algorithm (FiNTA). To the best of our knowledge, the FiNTA analyses more parameters than other computational tools designed for network analysis. These parameters include the filament length, the connectivity (also known as the coordination number, and the persistence length in combination with the angle distribution. Thus, this algorithm can be used to analyze any kind of network in grayscale two-dimensional (2D) images.

\section{MATERIALS AND METHODS}

\section{1 | Vectorial filament network-tracing algorithm}

The FiNTA is based on vectorial tracing of grayscale images. The tracing data generated by FiNTA consist of connected nodes which travel along the filaments. Since neither the node positions nor their connection lengths need to be constrained to the discrete positions of pixels, we call FiNTA "vectorial." Therefore, although binary images can be analyzed, there is no need to create binary images or pixel-based center lines prior to an analysis. To recognize the filaments in grayscale images, the FiNTA first identifies the directions of the filaments using image convolution with the Hessian matrix of the Gaussian kernels; thus, by validation of every pixel in terms of their intensities. ${ }^{20}$ This is followed by the generation and connection of nodes along the filaments, which results in the tracing lines (Figure 1A). Since this procedure differs from segmentation or pixel-based center lines, we compared the tracing results of FiNTA with DiameterJ and NeuronJ, respectively (Figure S1). It is possible to manually adjust the sensitivity of the method to any grayscale image by changing the input parameters, as described in Supporting Information (Table S1). For a more detailed description of the working principles and the mechanisms behind the algorithm, please see Supporting Information. The FiNTA identifies the filaments in a suitable time range; depending on the network complexity, the time range can be from seconds to minutes. It then automatically extracts the important network parameters. The algorithm is available on: https://github.com/SRaent/FiNTA.

\subsection{Extracted parameters}

The FiNTA provides information on at least eight of the most relevant parameters, which are: mesh hole size; circularity of each mesh hole; junction distance; filament density, filament length, connectivity of each unified junction, global angle distribution, and the persistence length.

The mesh hole size (MHS) is the measure of the network pore size, pore area, or hole size, or similar, and this is defined as the area of the pores or holes within the network (Figure 1B). The circularity is defined as $\mathrm{Ci}=4 \pi(M H S) / P^{2}$ , where $P$ is the perimeter of a mesh hole. Consequently, the circularity is a measure of the fractal dimension of the mesh holes. The filament density is the total network length divided by the total mesh hole area. Furthermore, the junction distance as the length of a filament between two junctions (Figure 1B). The filament length is the distance between nodes before reaching a defined break-off angle $\theta$ within two adjacent connections (Figure 1C). The number of filaments that are connected to a junction is described with the term connectivity, which in three-dimensional (3D) data analysis is often referred to as the coordination number. As a value of two represents a single filament, the minimum value of the connectivity is three, while the maximum value is unlimited. For instance, in actin networks, the connectivity rarely exceeds six, and these filaments typically have a mean connectivity of around 3.4. Within a user-defined unification distance FiNTA identifies junctions that are close to each other and considers them as one junction (Figure 1D). This is needed since junctions often have an expansion larger than the filament diameter, which forces FiNTA to identify two junctions instead of one for example. The global angle distribution is defined as the angles of the connections between the nodes relative to the image orientation measured counter clockwise, where the left edge of the image represents $0^{\circ}$, respectively. The worm-like chain model approximation is used to extract the persistence length. ${ }^{21}$ 
F I G URE 1 Graphical illustration of four parameters implemented in the filament network-tracing algorithm (FiNTA).

A, Algorithm routine to trace filaments vectorially. Starting with the original image, followed by kernels and angle analysis that leads to tracing lines finally. Filament thickness: 12 px. B, A single mesh hole with several junctions. $\mathrm{C}$, The filament length (FL) calculation. The break-off angle is $\theta$ (or smaller). The break-off angle assigned to the FiNTA is $\theta-180^{\circ}$ (as for all other angles), or larger. D, Implementation of the Connectivity condense the junctions along the unification distance shown as the thick blue line. In the upper example, within two nodes (blue squares) per filament no other junction (white circle) was identified by FiNTA. In the lower example, within three nodes per filament one other junction was identified. Since the unification distance was three (nodes) in this example, FiNTA summarizes both junctions to one leading to a connectivity of 5
(A)

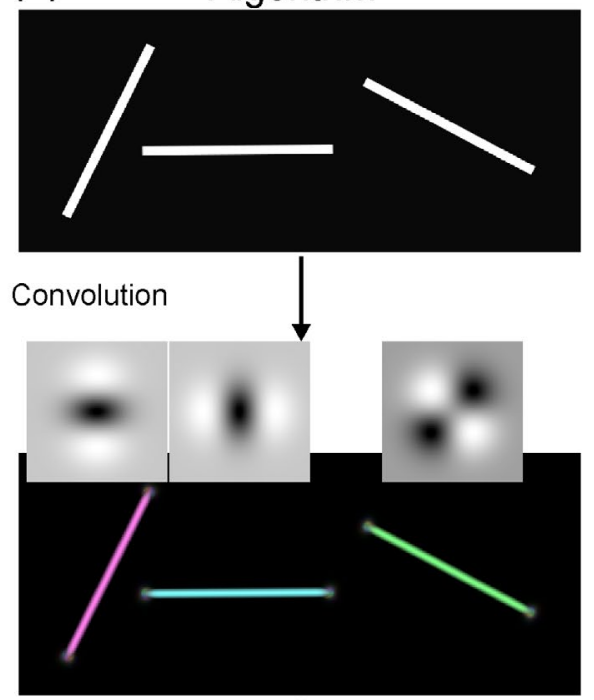

Filament angle analysis at node position

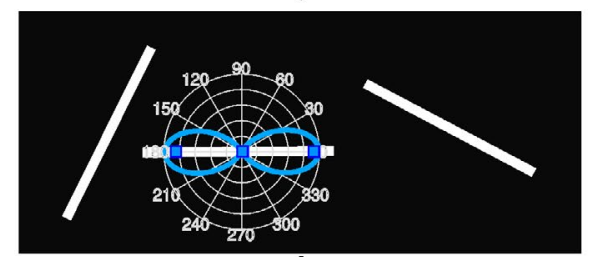

Addition of new nodes in analyzed direction

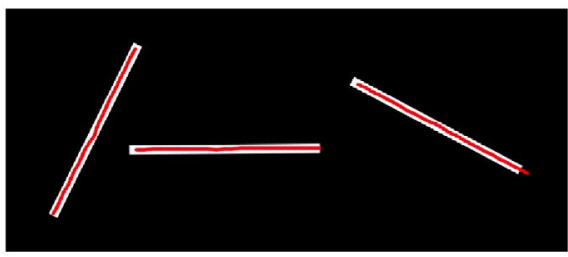

(B)

\section{Parameters}

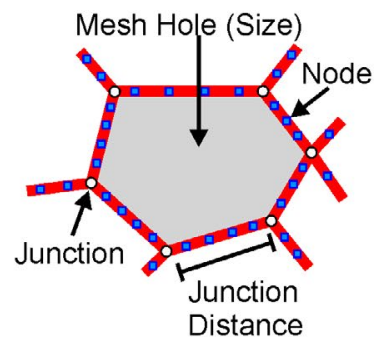

(C)

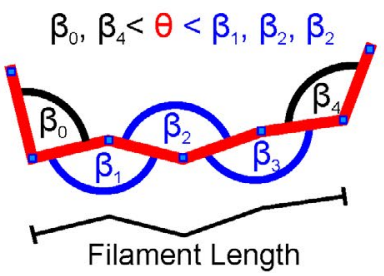

(D)
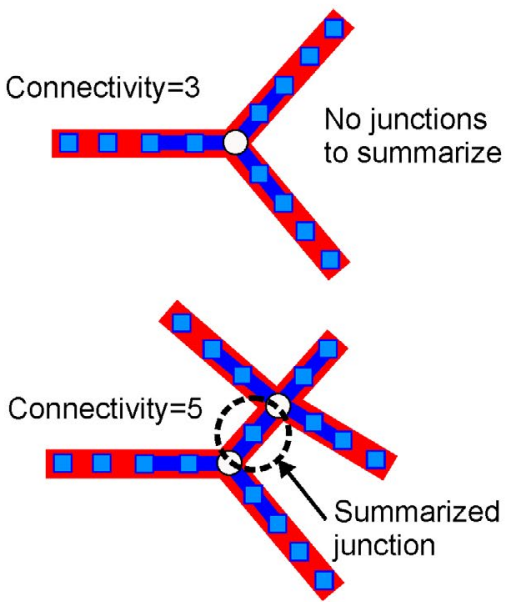

To demonstrate which parameters are easier to extract with the FiNTA, we analyzed three scanning electron microscopy images of structurally different actin microfilament networks in cells. These were taken over the nucleus, in the perinuclear area, and in a lamellipodium at the cell edge, as shown in Figure 2A. We thereby determined the density and structure of the actin microfilament network, and how it differed between the different subcellular regions, according to 18 different parameters (Figure 2B). This broad parameter space leads to a high flexibility of FiNTA compared to other algorithms. Therefore, extracted parameters that are identical to DiameterJ or NeuronJ are presented in Supporting Information (Table S2).

\section{3 | Biological sample preparations and imaging}

The preparation of the biological samples and the imaging details are in the Supporting Information.

\section{3 | RESULTS}

\section{1 | Filament density, filament angles, and mesh hole size}

The filament density is commonly calculated by determining the length of the total traced network, and dividing by the image size. To quantify the quality of this analysis, a realistic range of values of filament packing/densities were tracked that were within an acceptable error (10\% SD). An orthogonal grid of white filaments on a black background was digitally created. In this network, the number of filaments was then increased, from a filament density of $26 \%$ white and $74 \%$ black pixels, to $92 \%$ white and $8 \%$ black pixels, as shown in Figure 3A-D. The sizes and counts of the mesh holes in these images were then analyzed using the FiNTA, which were compared to the known sizes and counts of the designed mesh holes. The results with the FiNTA were similar to the known mesh hole sizes and counts (Figure 3E,F). This indicated that the FiNTA provided an accurate description of 

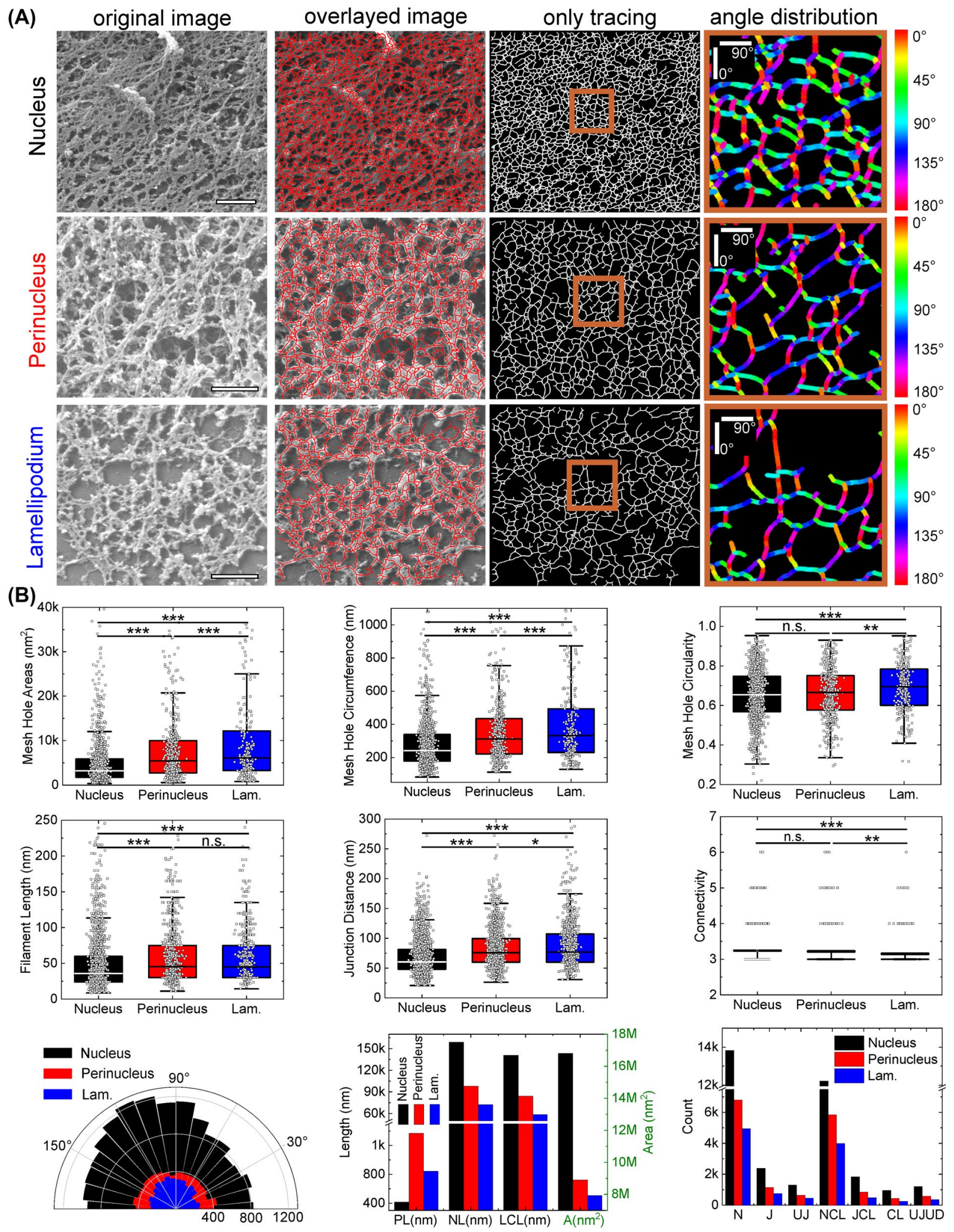

F I G U RE 2 Scanning electron microscopy examples and parameters. A, Representative actin images showing the tracing and angle distributions of three different cell regions of RPE1 cells. Scale bars and edge length of angle distribution images: $500 \mathrm{~nm}$. B, Quantification of 18 parameters extracted by the FiNTA. Lam., lamellipodium; PL, persistence length; NL, total network length (to calculate, eg, fiber density LCL, total network length of closed loops; A, total area of all loops; N, total number of nodes; J, total number of Junctions; UJ, total number of united junctions upon user defined unification distance used to calculate the connectivity; NCL, total number of nodes that contribute to closed loops; JCL, total number of Junctions that contribute to closed loops; CL, total number of closed loops; and UJUD, total number of united junctions of the network that only contain closed loops with the user-defined unification distance 
(A)

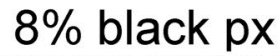

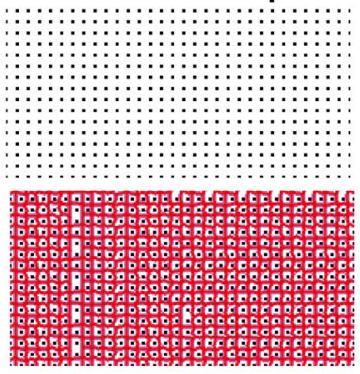

(E)

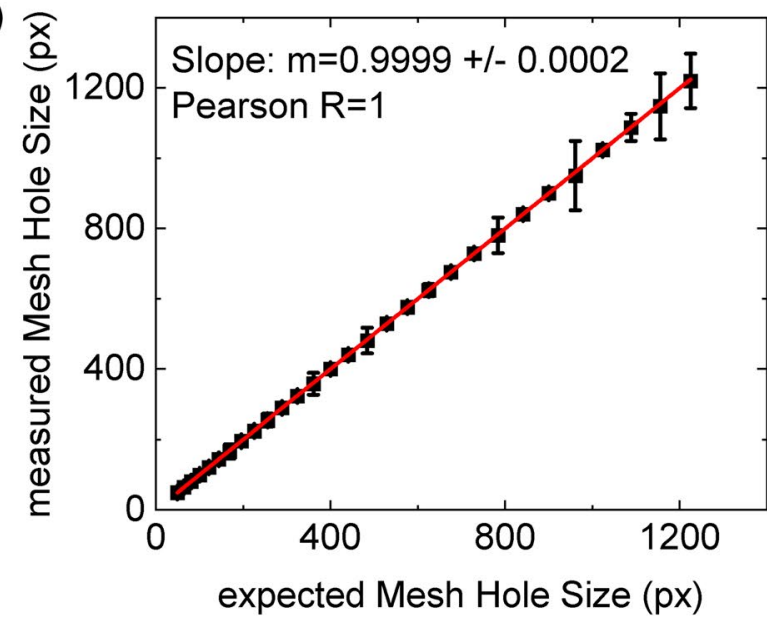

(G)

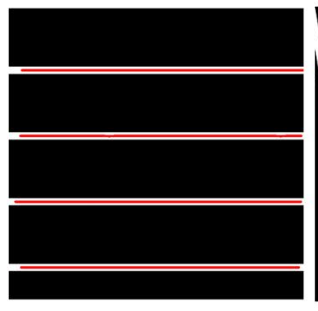

(B) $30 \%$ black px

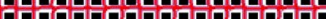

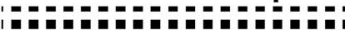

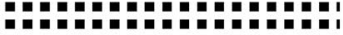

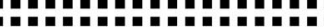

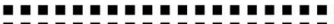

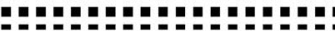
"

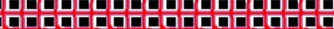

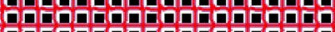

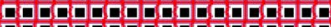

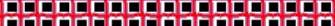

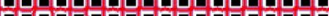

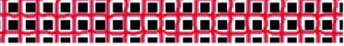

(C) $50 \%$ black px

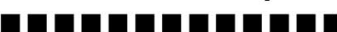

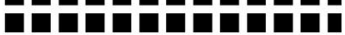

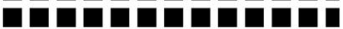

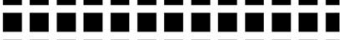
ㅁㅁㅁㅁㅁㅁ

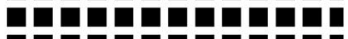

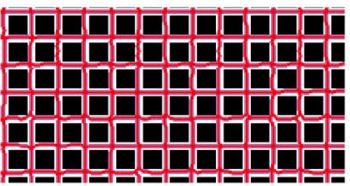

(D) $70 \%$ black $\mathrm{px}$

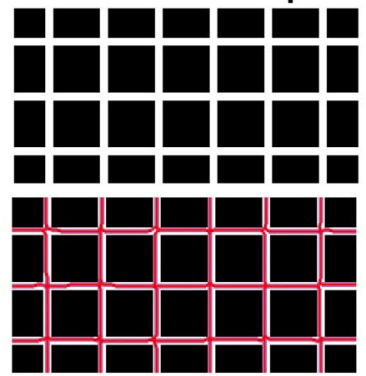

(F)
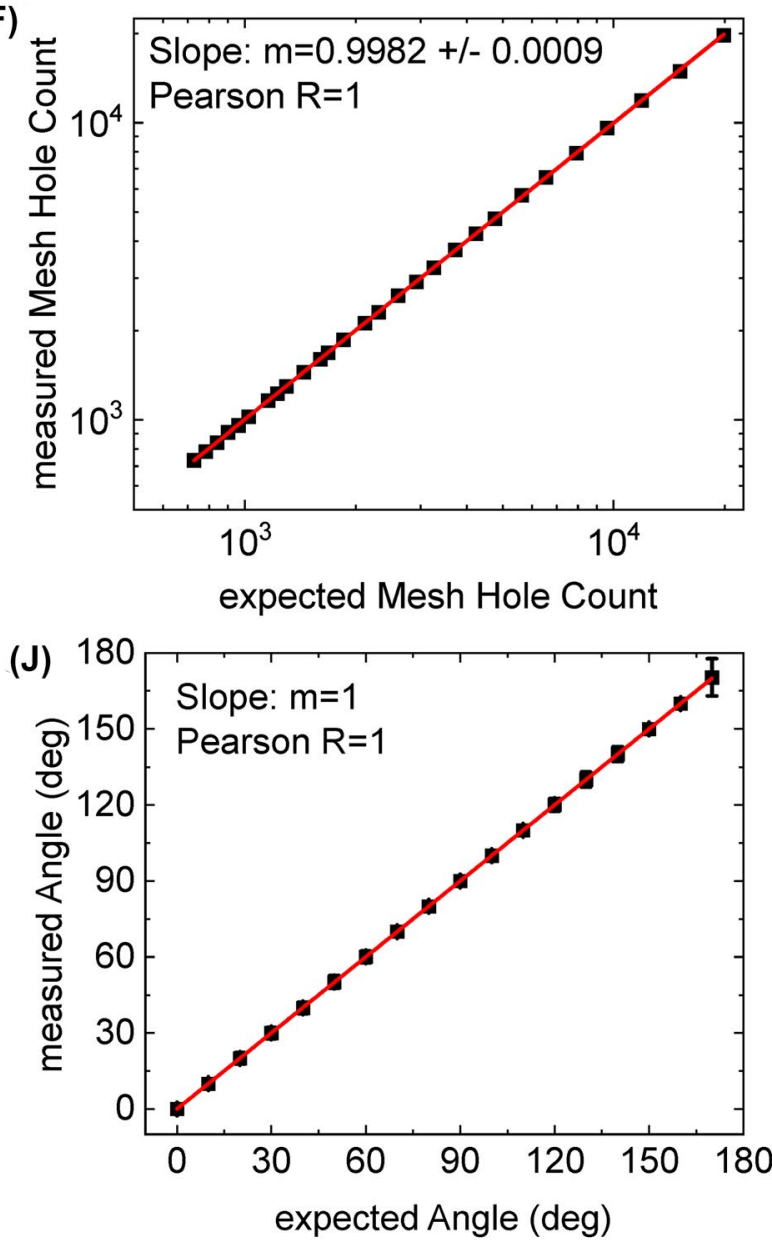

F I G U R E 3 Fiber density and orientation quality tests. A-D, Cropped regions of exemplary images with increasing black pixel fractions (decreasing filament density), with tracing results in the bottom half. Line thickness: 6 px. E, F, Linear approximations of expected vs measured mesh hole size (E) and mesh hole count (F). G-I, Exemplary images of different global filament angles with a line thickness of 27 px, that lead to accurate tracing lines. J, Linear approximation of expected vs measured angles

these porous and dense networks, with detection of up to $92 \%$ of the filaments.

To quantify the accuracy of the FiNTA analysis of the global filament angles, 18 images were created with filament angles from $0^{\circ}$ to $170^{\circ}$, in $10^{\circ}$ increments (Figure $3 \mathrm{G}-\mathrm{I}$ ). The following FiNTA analysis of the filaments angles was very similar to the expected values, with a Pearson's correlation $R=1$ (Figure 3J). This indicated that the FiNTA provided an accurate analysis of the global filament angles.

\subsection{Image resolution and noise level}

Image resolution and the signal-to-noise ratio govern the quality of the various imaging methods. We thus determined how these features of images influence the FiNTA tracing of networks. For this, test images were initially created with a filament density grid of $74 \%$ black. To recognize different network types, the FiNTA detection of different grid sizes was also tested by analysis of lines of six different sizes of pixels. 
For the signal-to-noise ratio, six digital noise levels were added, as infinite to zero signal-to-noise ratios, and the noise levels were calculated. The tracing quality of the FiNTA was then analyzed at these different signal-to-noise ratios by comparisons of the measured to the expected mesh hole size and count with the similarities expressed as percentage errors (Figure 4). Here, the higher the resolution of the image, the more noise was acceptable, and vice versa. Therefore, the FiNTA can trace images across a wide range of image resolution, and with various noise levels.

The typical signal-to-noise ratios of fluorescence and electron microscopy images of the cellular actin cortex are in the range of $5 \mathrm{~dB}$ to $15 \mathrm{~dB}$.

\section{3 | Connectivity}

The connectivity (also known as coordination number or branching number) is rarely included in algorithms for segmentationbased network analysis, and to the best of our knowledge, it has not been included in vectorial- tracing algorithms. Nonetheless, it is an essential parameter that is important to include and describe in any comprehensive analyses of networks. Therefore, the connectivity is also determined in the FiNTA.

To determine the efficiency of the connectivity measured by the FiNTA, four black and white 2D test images were artificially created where the only connectivity values were three, four, five, and six. As a connectivity of two represents a line, the minimum value of the Connectivity is three.
The connectivity measured on the original images with the FiNTA was then compared to the expected values, as shown in Figure 5A-D. These FiNTA values matched those expected (Figure 5E, F), and therefore, the FiNTA can measure all of the predominant connectivities within 2D networks.

\section{4 | Filament length}

In addition to the mesh hole size and connectivity, the filament length is a crucial parameter in the characterization of networks. To include this parameter in the FiNTA, a userdefined break-off angle was implemented to detect and define a filament as a single filament (see Figure 1C). This definition and implementation to FiNTA allows "one filament" to cross junctions identified by FiNTA. This is crucial since in a two dimensional image it is often not possible to differ between a physical junction and an overlay of filaments above each other. Consequently, we implemented the break-off angle in order to be not limited by the identification of junctions. Even visual inspection of network images does rarely allow the identification of the beginning or end of a filament. Therefore, the filament length calculated by FiNTA is most likely not the real filament length, but a filament length close to the one that is identified by visual inspection.

To determine the accuracy of this filament length tracing approach, the artificially created test images for the connectivity were modified to show a homogenous distribution of filament lengths by removing the filaments at the

(A)

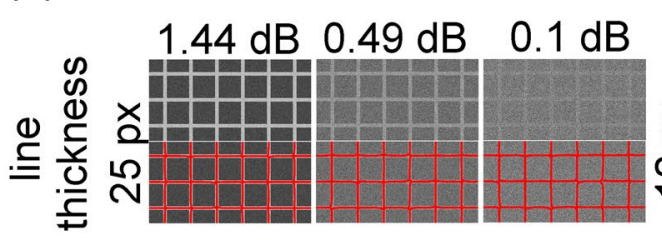

Signal to Noise Ratio
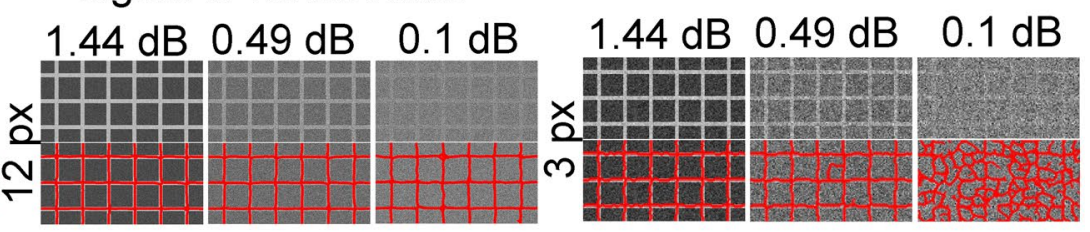

(B)

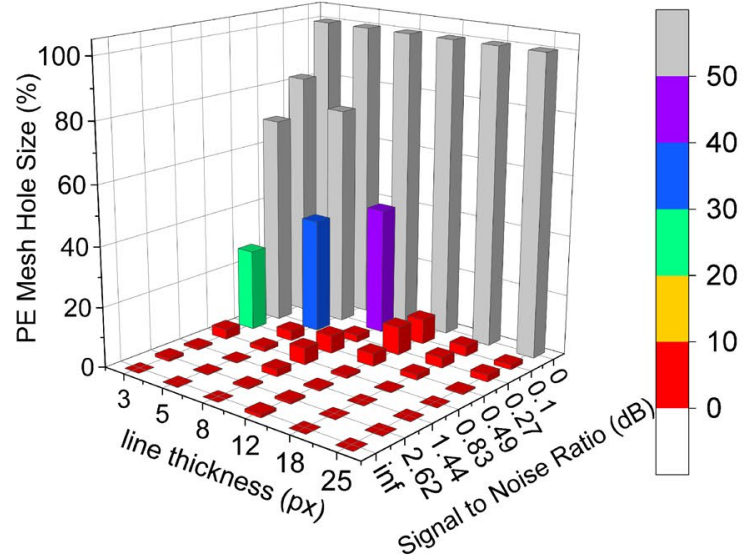

(C)

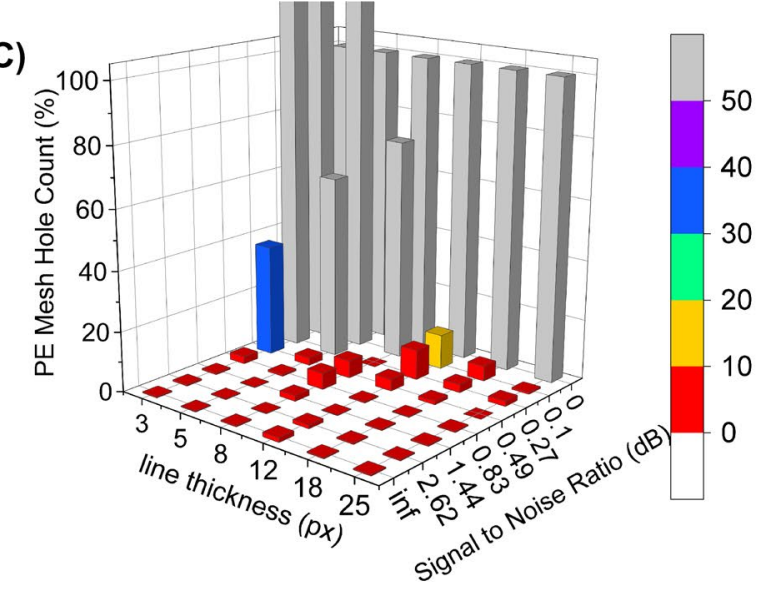

F I G U RE 4 Mesh hole size by varying signal-to-noise ratio and line thickness. A, Exemplary images of traced mesh holes by varying signalto-noise ratio and line thickness. B, Percentage errors (PE) of expected mesh hole size and count vs measured mesh hole size (B) and count (C), with dependence on signal-to-noise ratio and line thickness 
(A)

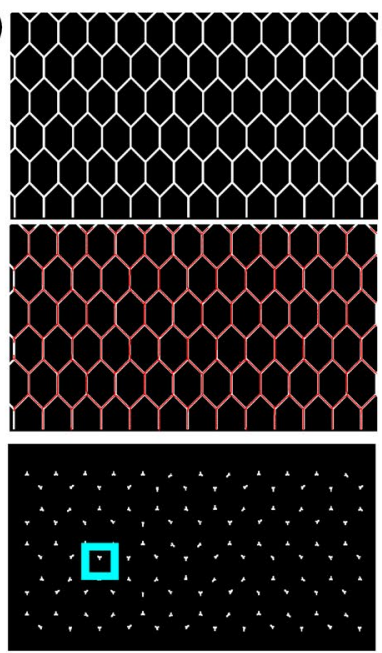

(B)

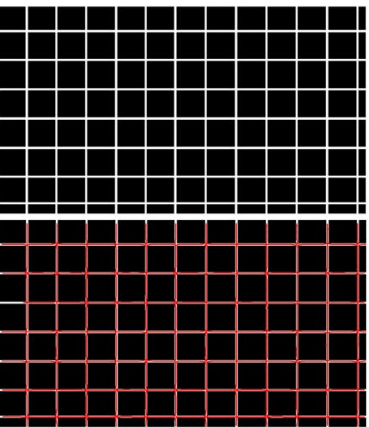

(E)

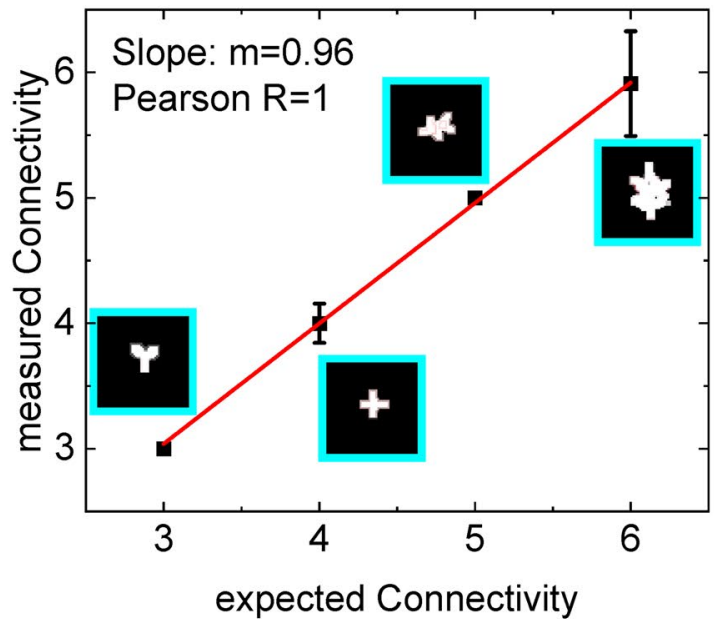

(G)

Expected Filament Length: $71 \mathrm{px}$

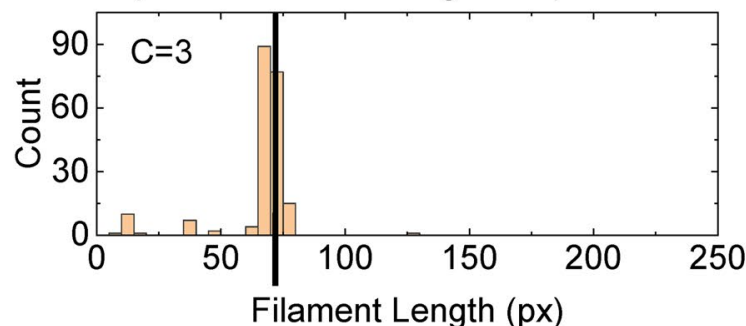

Expected Filament Length: 93 px

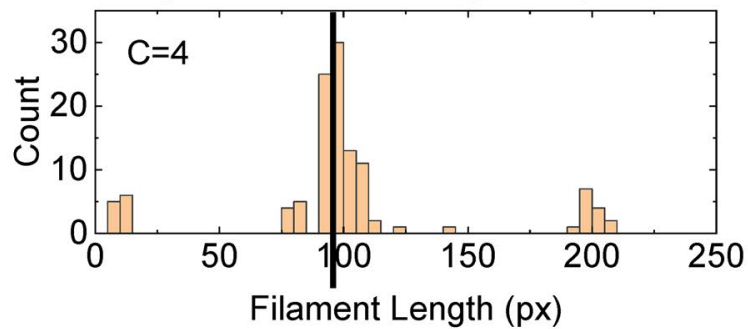

$+$
(C)

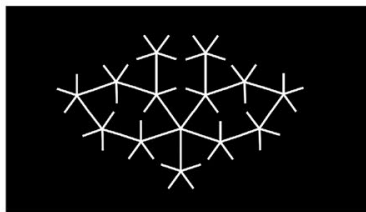

(D)
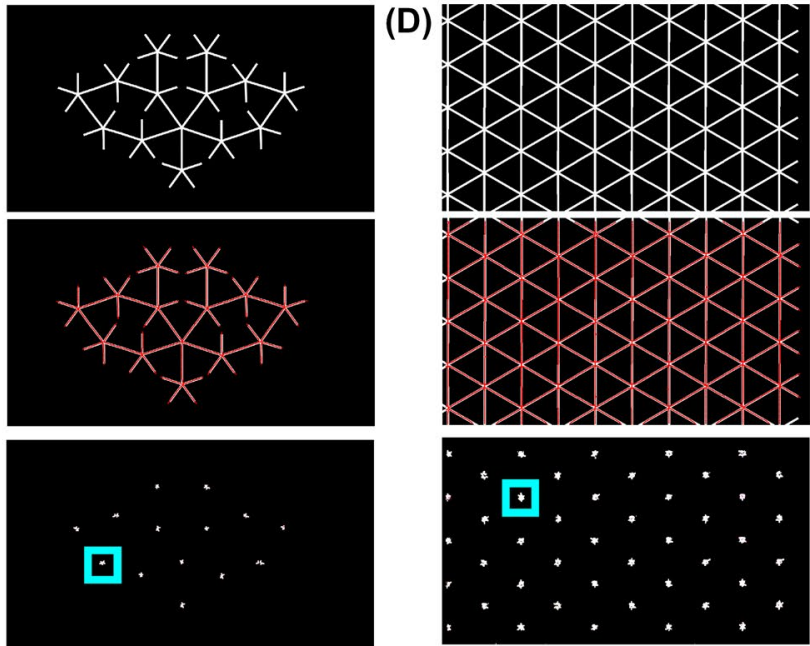

(F)

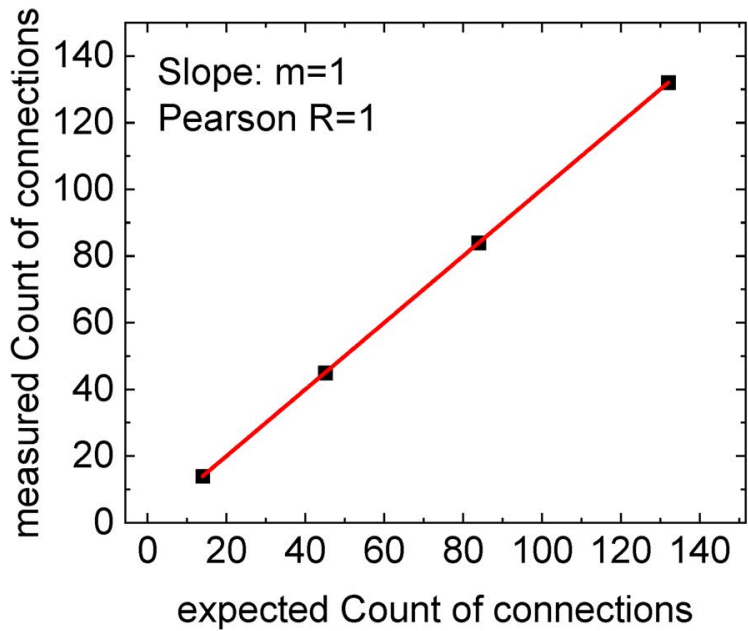

Expected Filament Lengths: 78 px and 140 px

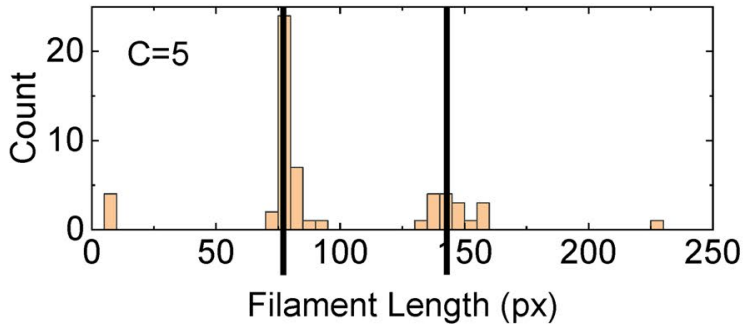

Expected Filament Length: $140 \mathrm{px}$

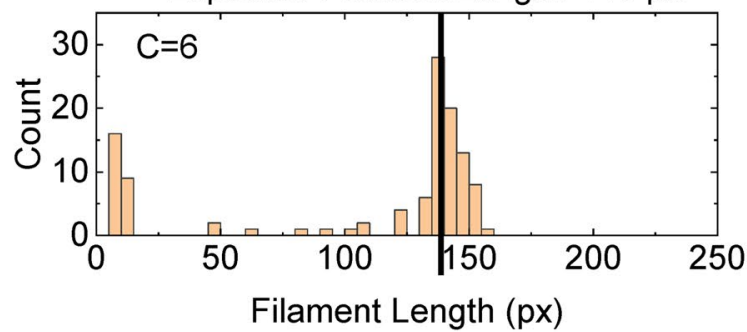

F I G U R E 5 Connectivity and Filament Length tests. A-D, Exemplary images with discretely increasing connectivities as three (A), four (B), five (C), and six (D), as are traced in red (second row), and with branches/junctions extracted (third row). Junction connectivity was set to 6 (ie, all junctions that can be connected by 6 or less steps are unified to one junction). Line thickness: 9 px. E, F, Linear approximations of expected vs measured Connectivity (insets show corresponding third rows of (A-D)) (E) and counts of branches/connections (F). Red lines show the linear approximation. G, Filament length histograms of images (A-D) corresponding to the four connectivities from three to six. The break-off angle was set to $\sim 17^{\circ}(0.3 \mathrm{rad})$. Black vertical lines represent the expected filament length 
edges of the images. This resulted in data with very similar filament lengths, as shown in Figure 5G for a break-off angle of $\sim 17^{\circ}(0.3 \mathrm{rad})$, where all of the filament lengths were within an accurate range of the expected lengths. However, the FiNTA also identified artificial filaments that were significantly shorter than those expected, and were localized to the branching/connection zones. Such very short filaments can be eliminated easily using a threshold value. We also performed a more detailed test of the filament lengths and their dependence on the break-off angle, as presented in Figure S2.

\subsection{Other extracted parameters}

As the mesh hole size, connectivity, and filament lengths should be the most challenging parameters to implement, we mainly focused on testing these parameters in detail. Additional parameters that can be calculated based on the mesh hole size and from the tracing per se were also tested. The filament density can be calculated from the length of the total traced network divided by the image size. The junction distance (so-called fiber length in DiameterJ) was calculated forward from the known junction positions. The connectivities from the FiNTA were very accurate for both the quality and the counts, and thus the reliability of the analysis of junction distance was also accurate, as for the connectivity. The circularity is the fractal dimension of the mesh holes, and this was defined as proportional to the mesh hole size divided by the perimeter squared. The reliability of the analysis of circularity is therefore strongly depending on the mesh hole size, which was shown to be very accurate in Section 3.1.

\section{6 | Tracing accuracy}

The accuracy of the tracing determines the quality of all of the parameters that are extracted from any tracing software. To determine the tracing accuracy with the FiNTA, we analyzed electron microscopy images of actin filaments and a fluorescence confocal microscopy image of microtubules (Figure 6). For the quality of the analysis, the filaments traced with the FiNTA were then also traced manually in the same images. This thus determined the relative levels of false-negative and false-positive signals obtained using the FiNTA, as the relative proportions of nontraced filaments and nonexisting filaments traced, respectively.

For determination of the levels of false-negative signals, the relative lengths of nontraced filaments were calculated by dividing these by the total network length traced by hand including the nontraced filaments. For determination of the levels of false-positive signals, the proportion of false-positive filaments were defined according to the total network length traced by hand. On this basis, the FiNTA tracing for the electron microscopy images showed $8.55 \%$ false-negative filaments and $1.54 \%$ false-positive filaments. For the fluorescence image FiNTA tracing, there were 9.72\% false-negative filaments, and $1.42 \%$ false-positive filaments. Therefore, the false-positive rates can be considered as negligible, and the false-negative rates are $<10 \%$, which can be considered an acceptable value. The falsenegative filaments arise because the FiNTA does not trace up to the edge of the image, to avoid any distortion of the mesh hole size. In the central parts of the images, almost all of the filaments that are not traced (ie, the false negatives) arise where the angle between two filaments is small.
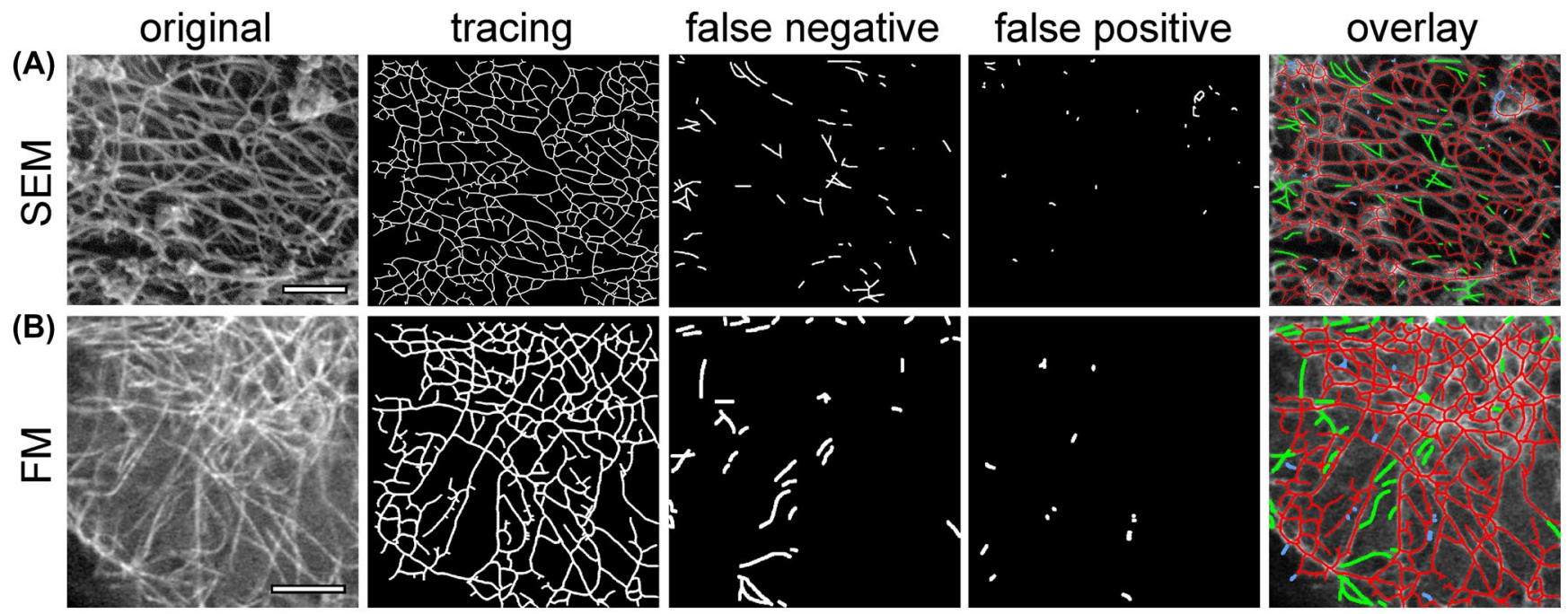

F I G URE 6 Tracing error tests. Representative images of actin filaments obtained using scanning electron microscopy (A; SEM) and of microtubules obtained using confocal fluorescence microscopy (B; FM), as traced with the FiNTA and compared to hand tracing. Nontraced fibers (false negative) and nonexisting filaments (false positive) that were traced are shown ( $2 \times$ fiber thickness of original tracing). Overlay of the correct tracing (red), false negatives (green), and false positives (blue) shown in the last column. Scale bars: $200 \mathrm{~nm}(\mathrm{~A}) ; 5 \mu \mathrm{m}$ (B) 


\subsection{Network separation}

It is challenging to draw conclusions about spatial distributions in three dimensions from 2D images of 3D networks, both manually and automatically. For example, it is usually not possible to distinguish between a real junction and an overlay of two filaments. However, the FiNTA can reduce these events to a minimum. Here, this is based on the effect whereby the filaments closer to the camera (ie, at a higher level within the network) are brighter than the filaments that are further away from the camera (ie, at a lower level within the network). This is true for images from both electron microscopy and fluorescence microscopy. For this, the option was implemented to preserve traced nodes within a specified grayscale interval only, which can be chosen by the user. This approach can also be used to avoid over-exposed and under-exposed regions of a network.

To assess the quality of this approach, three different images were analyzed at high and low intensity levels (Figure 7). For electron microscopy images of actin networks, the high intensity areas, referred to as ruffles, provide no information about the network due to their overexposure. It might therefore be of interest to exclude these high intensity regions from the tracing, which is what the FiNTA does (Figure 7A).
To determine the quality of the FiNTA for other types of networks, an electron microscopy image of the hexagonal structure of a diatom alga was analyzed. The thresholding of the intensity values allowed the FiNTA to discern the hexagonal structures from the underlying network (Figure 7B). Furthermore, in fluorescence images of actin, the FiNTA can differentiate between high intensities (more fluorescein proteins) and low intensities (less fluorescein proteins) (Figure 7C). This allows the user to focus on the subcellular regions of a network, which can be useful if the user is interested in subregions, or parts of the intensity distribution within an image. Therefore, such intensity thresholding is a powerful method to separate networks for their detailed investigation.

\subsection{Tracing of cytoskeletal networks imaged by electron and fluorescence microscopy}

To demonstrate the wide range of FiNTA applications, images of different cytoskeletal filaments were traced that were obtained by electron microscopy (Figure 2) and using different variants of fluorescence microscopy (Figure 8).

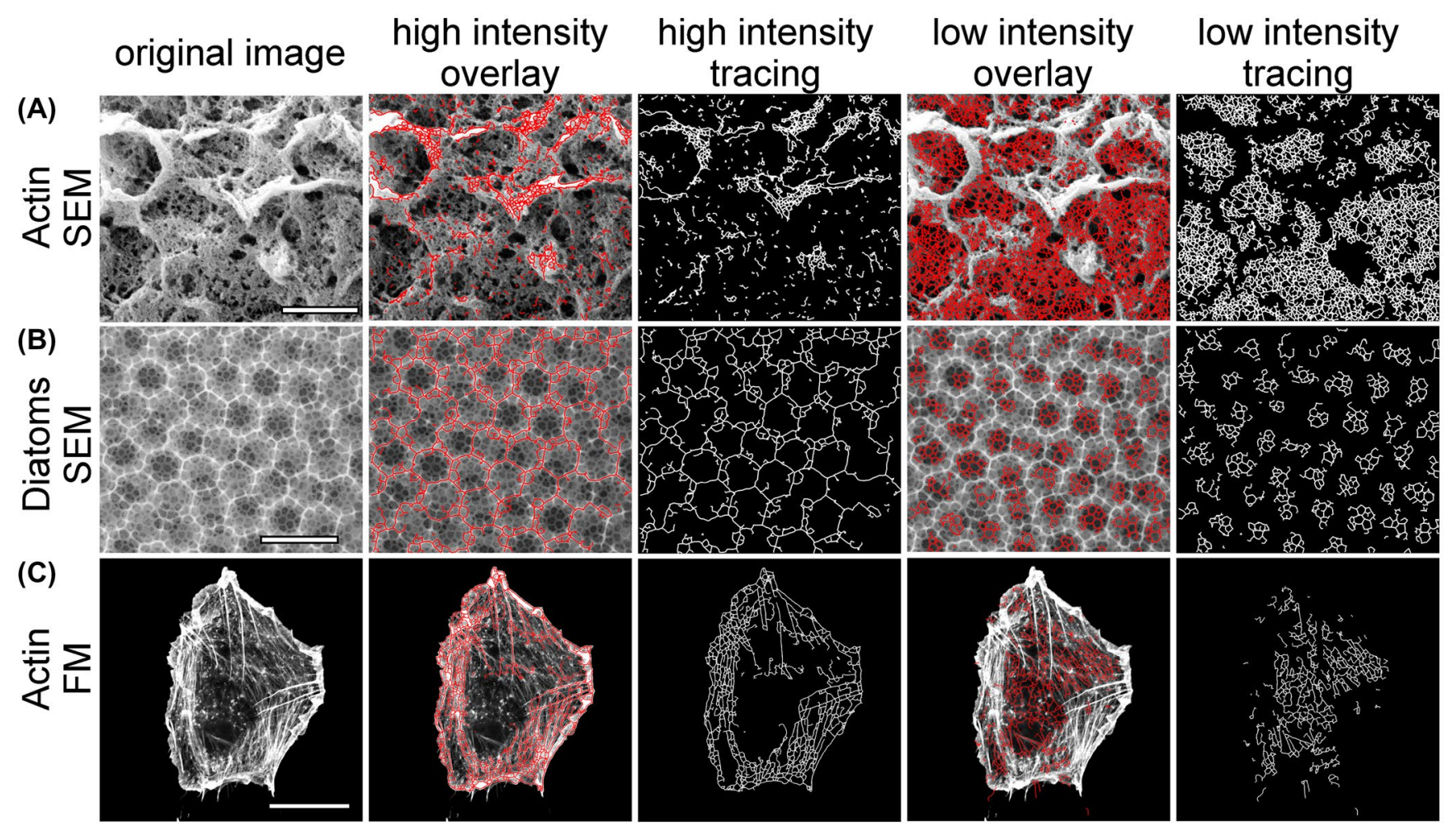

F I G URE 7 Network separation by intensity thresholding. Representative images for scanning electron microscopy (SEM) of actin (A) and a diatom (B) and fluorescence microscopy (FM) of actin (C), used to separate the networks by intensity. A, B, As used to eliminate unwanted areas (A, actin: high intensity) and analyze the remaining network (low intensity), or to separate a superior (B, diatom, high intensity) and an inferior (low intensity) network. C, As used to separate between high and low intensity signals that represent high and low amounts of fluorescent actin molecules. Scale bars: $1 \mu \mathrm{m}(\mathrm{A}), 4 \mu \mathrm{m}$ (B), $20 \mu \mathrm{m}$ (C) 

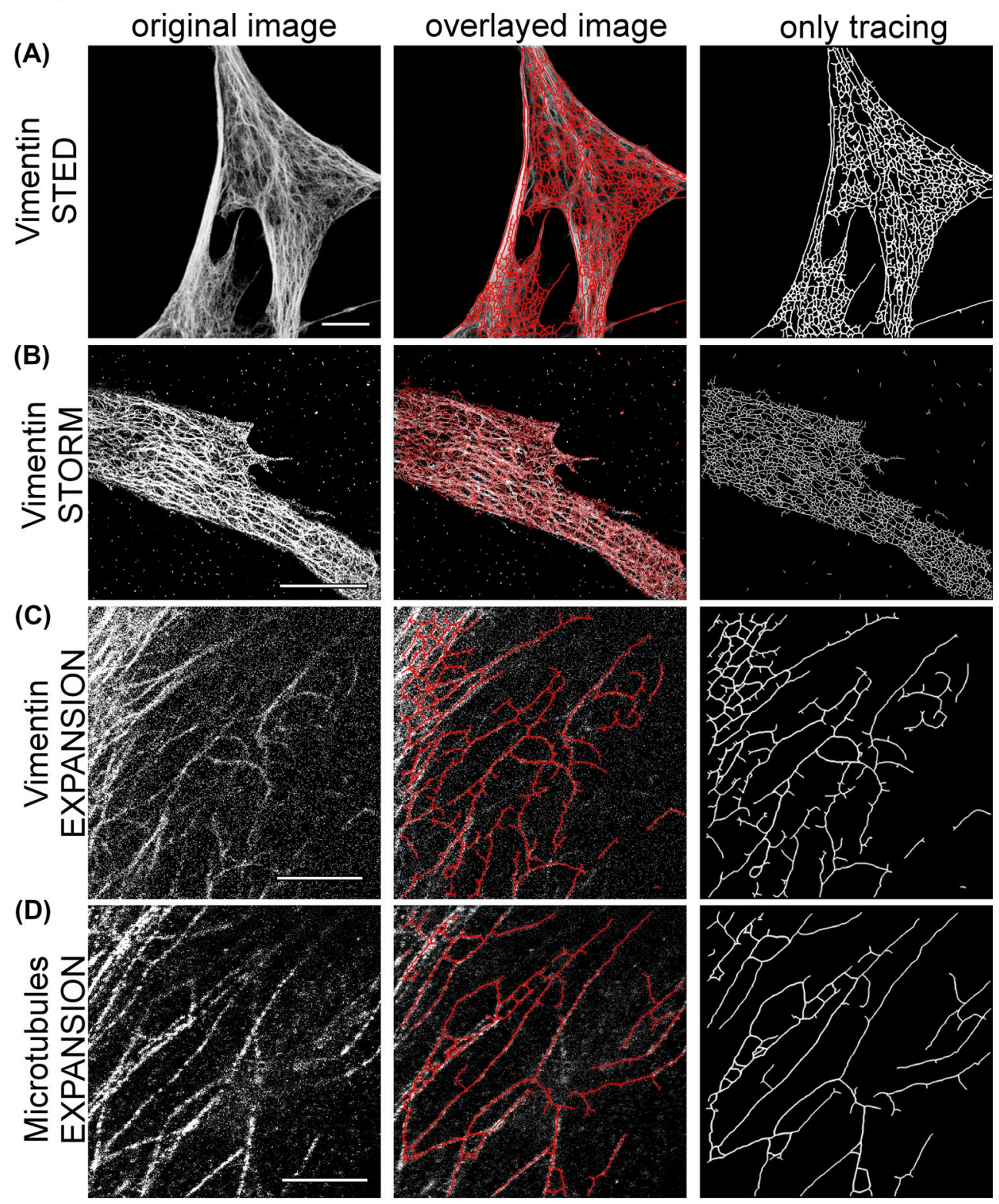

F I G U R E 8 Representative images and exemplary traced fluorescence super-resolution networks. Vimentin was imaged and traced (as indicated) using stimulated emission depletion (STED) microscopy (A), 3D stochastic optical reconstruction microscopy (STORM) (B) and Expansion microscopy (C). D, Identical image section of cell vimentin in (C) is shown for microtubules in (D). Overlayed image: overlay of original and traced images. Scale bars: $10 \mu \mathrm{m}$ (A, B) (expansion factor, 4.5); $2.38 \mu \mathrm{m}$ (C, D)

We showed in Section 3.7 that the FiNTA can be used to analyze actin microfilament cytoskeletal networks imaged by confocal microscopy (Figure 7C). However, the resolution of confocal microscopy is often not sufficient to determine the fine structure of cytoskeletal networks, as more recently, super-resolution techniques are being increasingly used. To provide a useful tool for such studies, the reliability of the FiNTA was analyzed using images from superresolution microscopy. Images of the intermediate filament protein vimentin were analyzed for various cell types that were provided by three distinct super-resolution microscopy techniques that are based on different approaches and principles: stimulated emission depletion (STED) microscopy; stochastic optical reconstruction microscopy (STORM); and expansion microscopy. STED microscopy is a type of confocal microscopy where a homogenous signal along filaments is recorded. In contrast, STORM and expansion microscopy are techniques that create a pointillismlike signal along filaments. In STORM microscopy, this is achieved by the imaging technique itself, whereas in expansion microscopy, the structure under investigation is physically ruptured at the nanoscale level. These vimentin network images using each of these super-resolution techniques were indeed accurately traced by the FiNTA 
(Figure 8A-C). This included the pointillism-like signals of STORM and expansion microscopy, as shown in the detailed pointillism test in Figure S3, and more indirectly in Figure 4. To further confirm this, Figure 8D shows the FiNTA tracing of a microtubule meshwork obtained using expansion microscopy. Consequently, we can conclude that the FiNTA can also correctly trace networks in images using super-resolution fluorescence imaging techniques.

As indicated in Section 2.2, the FiNTA can trace actin microfilament networks obtained using electron microscopy. To determine whether the FiNTA can also be used for electron microcopy images of other structures, we additionally investigated mushroom braid, eggshell, foam, and diatoms. With the exception of the foam, which showed high heterogeneity for the filament thickness and brightness, the FiNTA provided accurate tracing of these networks (Figure S5). Consequently, FiNTA is scale-independent and could be used for fundamentally different questions, such as cell-cell junctions (eg cell monolayers stained for E-cadherin) or even network-spanning organism (eg, Physarum polycephalum).

To further validate the FiNTA, other images were analyzed, including a picture of sticks of spaghetti obtained using a reflex camera, and different painted, biological, and written examples, which again resulted in consistently accurate tracing (Figure S6).

\section{4 | DISCUSSION}

In this study, we present the powerful, rapid, user-friendly, open-source vectorial FiNTA for grayscale images of different filament types and across a large variety of scales. This is shown by our analysis of the intracellular nanoscale actin microfilaments and vimentin cytoskeleton, and of other network forms using various imaging techniques.

A lot of the available software and plugins for analysis of networks require the raw data images in grayscale to be converted into binary images prior to analysis, such as the ImageJ plugin DiameterJ. The quality of the images will thereby greatly impact on the recognition of filaments by the software. With the FiNTA, grayscale images can be analyzed, which thus overcomes this problem.

The FiNTA allows analysis of the common network parameters, such as mesh hole size, circularity, junction distance, filament density, and others. Moreover, it provides a high level of quantification of challenging parameters, such as connectivity and filament length. The FiNTA can also be used to separate subnetworks in single images, through intensity thresholding. It has a high tolerance in terms of filament density and noise. Moreover, the FiNTA delivers good results regardless of the imaging technique used (eg, fluorescence microscopy, scanning electron microscopy, photographs), and of the substance investigated.
One limitation of the FiNTA relates to networks with small angles between the filaments. The FiNTA was also implemented for homogenous filament thickness. Although the FiNTA can be used where there are small variations in filament thickness, significant heterogeneity in filament thickness across a network can lead to nonideal tracing. Finally, heterogeneous intensity distributions along filaments can result in an increased false tracing by the FiNTA. In such cases, segmentation-based algorithms might provide more accurate tracing.

In summary, the FiNTA is an algorithm that can provide great benefits across a wide variety of scientific fields that involve analysis of images of networks.

\section{ACKNOWLEDGMENT}

The authors thank the Leibniz Institute for New Materials (INM), Saarland University, and the DFG (CRC 1027 (A10)) for financial support. The authors also thank the Weston Park Cancer Centre (University of Sheffield) the Fundação para a Ciência e a Tecnologia (FCT), the Portuguese Government (PEst-OE/QUI/UI0674/2013) and the Agência Regional para o Desenvolvimento da Investigaçaõ Tecnologia e Inovação (ARDITI), M1420-01-0145-FEDER-000005-Centro de Química da Madeira-CQM (Madeira 14-20). We thank Gaudenz Danuser (Texas, USA) for the hTERT-RPE1 cells stably expressing mEmerald-vimentin and mTagRFPt- $\alpha$ tubulin, Matthieu Piel (Paris, France) for the hTERT-RPE1 cells stably expressing mCherry LifeAct, and William C. Hahn (Harvard Medical School, USA) for the BJ cell line. We thank Ana Lopez and Christa Walther, University of Sheffield, UK, for excellent technical support. We thank Guillaume Charras (London Centre of Nanotechnology, UK) for excellent ideas about the parameters extracted by the FiNTA. Universität des Saarlandes (US), Open access funding enabled and organized by Projekt DEAL

\section{CONFLICT OF INTEREST}

The authors declare that they have no conflicts of interest.

\section{AUTHOR CONTRIBUTIONS}

D.A.D. Flormann, M. Schu, and E. Terriac designed the study. D.A.D. Flormann and F. Lautenschläger supervised the work. M. Schu developed the algorithm. D.A.D. Flormann and M. Schu tested the algorithm. D.A.D. Flormann and M. Koch prepared the samples and provided the electron microscopy images, which were obtained at the Leibniz Institute for New Materials (INM), Saarbruecken, Germany. A.K.B. Gad prepared samples and provided the STORM images, which were obtained at The Wolfson Light Microscopy Facility, University of Sheffield, UK. D. Thalla prepared samples and provided fluorescent STED images of vimentin, which were obtained at the Leibniz Institute for New Materials (INM), Saarbruecken, Germany. L. Kainka prepared samples and provided confocal 
images of microtubules, which were obtained at the Leibniz Institute for New Materials (INM), Saarbruecken, Germany. D.A.D. Flormann prepared samples and provided Expansion microscopy images of microtubules and vimentin, which were obtained at the Leibniz Institute for New Materials (INM), Saarbruecken, Germany. D.A.D. Flormann, A.K.B. Gad, and F. Lautenschläger wrote and revised the manuscript.

\section{REFERENCES}

1. Xia S, Lim YB, Zhang Z, et al. Nanoscale architecture of the cortical actin cytoskeleton in embryonic stem cells. Cell Rep. J. 2019;28(5):1251-1267.e7.

2. Zemel A, Rehfeldt F, Brown AE, Discher DE, Safran SA. Optimal matrix rigidity for stress fiber polarization in stem cells. Nat Phys. 2010;6(6):468-473.

3. Bovellan M, Romeo Y, Biro M, et al. Cellular control of cortical actin nucleation. Curr Biol. 2014;24(14):1628-1635.

4. Chugh P, Paluch EK. The actin cortex at a glance. J Cell Sci. 2018;131(14):jcs186254.

5. Nasrollahi S, Banerjee S, Qayum B, Banerjee P, Pathak A. Nanoscale matrix topography influences microscale cell motility through adhesions, actin organization, and cell shape. ACS Biomat Sci Engineer. 2017;3(11):2980-2986.

6. Maiuri P, Rupprecht J-F, Wieser S, et al. Actin flows mediate a universal coupling between cell speed and cell persistence. Cell. 2015;161(2):374-386.

7. Yamaguchi H, Condeelis J. Regulation of the actin cytoskeleton in cancer cell migration and invasion. Biochim Biophys Acta. 2007;1773(5):642-652.

8. Hall A. The cytoskeleton and cancer. Cancer Metast Rev. 2009;28(1-2):5-14.

9. Cordes A, Witt H, Gallemí-Pérez A, et al. Prestress and area compressibility of actin cortices determine the viscoelastic response of living cells. Phys Rev Lett. 2020;125(6):068101.

10. Hecht FM, Rheinlaender J, Schierbaum N, Goldmann WH, Fabry B, Schaffer TE. Imaging viscoelastic properties of live cells by AFM: power-law rheology on the nanoscale. Soft Matter. 2015;11(23):4584-4591.

11. Svitkina TM. Actin cell cortex: structure and molecular organization. Trends Cell Biol. 2020;30(7):556-565.
12. Gardel ML, Shin JH, MacKintosh FC, Mahadevan L, Matsudaira P, Weitz DA. Elastic behavior of cross-linked and bundled actin networks. Science. 2004;304(5675):1301-1305.

13. Chugh P, Clark AG, Smith MB, et al. Actin cortex architecture regulates cell surface tension. Nat Cell Biol. 2017;19(6):689-697.

14. Chen F, Tillberg PW, Boyden ES. Optical imaging. Expansion microscopy. Science. 2015;347(6221):543-548.

15. Svitkina TM. Platinum replica electron microscopy: imaging the cytoskeleton globally and locally. Int J Biochem Cell Biol. 2017;86:37-41.

16. Chikina AS, Svitkina TM, Alexandrova AY. Time-resolved ultrastructure of the cortical actin cytoskeleton in dynamic membrane blebs. J Cell Biol. 2019;218(2):445-454.

17. Hotaling NA, Bharti K, Kriel H, Simon CG. DiameterJ: a validated open source nanofiber diameter measurement tool. Biomaterials. 2015;61:327-338

18. Meijering E, Jacob M, Sarria JC, Steiner P, Hirling H, Unser M. Design and validation of a tool for neurite tracing and analysis in fluorescence microscopy images. Cytometry A. 2004;58(2):167-176.

19. Xu T, Vavylonis D, Tsai F-C, et al. SOAX: a software for quantification of 3D biopolymer networks. Sci Rep. 2015;5:9081.

20. Su R, Sun C, Pham TD. Junction detection for linear structures based on Hessian, correlation and shape information. Pattern Recogn. 2012;45(10):3695-3706.

21. Smith MB, Li H, Shen T, Huang X, Yusuf E, Vavylonis D. Segmentation and tracking of cytoskeletal filaments using open active contours. Cytoskeleton. 2010;67(11):693-705.

\section{SUPPORTING INFORMATION}

Additional Supporting Information may be found online in the Supporting Information section.

How to cite this article: Flormann DAD, Schu M, Terriac E, et al. A novel universal algorithm for filament network tracing and cytoskeleton analysis. The FASEB Journal. 2021;35:e21582. https://doi. org/10.1096/fj.202100048R 